\title{
SAÏD BENAYADI \\ Structure of perfect Lie algebras without center and outer derivations
}

Annales de la faculté des sciences de Toulouse $6^{e}$ série, tome $5, \mathrm{n}^{\circ} 2$ (1996), p. 203-231

<http://www.numdam.org/item?id=AFST_1996_6_5_2_203_0>

(C) Université Paul Sabatier, 1996, tous droits réservés.

L'accès aux archives de la revue «Annales de la faculté des sciences de Toulouse » (http://picard.ups-tlse.fr/ annales/) implique l'accord avec les conditions générales d'utilisation (http://www.numdam.org/conditions). Toute utilisation commerciale ou impression systématique est constitutive d'une infraction pénale. Toute copie ou impression de ce fichier doit contenir la présente mention de copyright.

\section{Numdam}

Article numérisé dans le cadre du programme

Numérisation de documents anciens mathématiques

http://www.numdam.org/ 


\title{
Structure of perfect Lie algebras without center and outer derivations ${ }^{(*)}$
}

\author{
SAÏD BENAYAdi ${ }^{(1)}$
}

RÉSUMÉ. - On montre que certaines propriétés classiques des algèbres de Lie semi-simples restent vraies pour les algèbres de Lie $g$ qui vérifient $[g, g]=g, \operatorname{Der}(g)=\operatorname{ad}(g), Z(g)=\{0\}$, que nous appellerons les algèbres de Lie sympathiques.

On construit une algèbre de Lie sympathique non semi-simple de dimension 25 . Cette algèbre de Lie est la plus petite algèbre de Lie sympathique non semi-simple connue à ce jour.

Si $g$ est une algèbre de Lie, on montre que $g$ contient un plus grand idéal sympathique $M$, et qu'il existe un idéal résoluble de $g$ noté $P(g)$ qui est le plus grand idéal résoluble $I$ de $g$ tel que $I \cap M=\{0\}$. Et on montre l'existence d'une sous-algèbre de Lie sympathique $m$ telle que $g=m \oplus P(g)$, et $g$ est sympathique si et seulement si $P(g)=\{0\}$.

On étudie les idéaux $I$ d'une algèbre de Lie $g$ tel que $g / I$ est sympathique.

Abstract. - We show that some classical properties of semi-simple Lie algebras are still valid for pertect Lie algebras $g$ without center and outer derivations (i.e $g$ satisfies $[g, g]=g, \operatorname{Der}(g)=\operatorname{ad}(g), Z(g)=\{0\}$ ). Let us call them sympathetic Lie algebras.

We construct the smallest non semi-simple Lie algebra (of dimension 25) known until now.

If $g$ a Lie algebra, then we show that $g$ contains a greatest sympathetic ideal $M$, and there exists a solvable ideal of $g$ denoted $P(g)$ which is the greatest among the solvable ideals $I$ of $g$ for which $I \cap M=\{0\}$. And we show that there exists a sympathetic subalgebra $m$ of $g$ such that $g=m \oplus P(g)$; and $g$ is a sympathetic Lie algebra if and only if $P(g)=\{0\}$.

We study the ideals $I$ of a Lie algebra $g$ such that $g / I$ is a sympathetic Lie algebra.

(*) Reçu le 2 mars 1994

This work has been taken from my doctoral dissertation (février 1993)

(1) Laboratoire de Physique Mathématique, Université de Bourgogne, B.P. 138, F-21004 Dijon Cedex (France) and current address : Département de Mathématiques, Université de Metz, URA CNRS 399, Ile-de-Saulcy, F-57045 Metz Cedex (France) 


\section{Notations and definitions}

Let $g$ be a finite dimensional Lie algebra over $\mathbf{K}=\mathbb{R}$ or $\mathbb{C}$. Let $\operatorname{Der}(g)$, $\operatorname{ad}(g), Z(g), R(g),\left(D^{n} g\right)_{n>0},\left(C^{n} g\right)_{n>1}$, denote respectively the algebra of derivations of $g$, the ideal of inner derivations of $g$, the center of $g$, the radical of $g$, the derived series of $g$, the descending central series of $g$.

(i) A Lie algebra $g$ is perfect if $g=[g, g]$.

(ii) A Lie algebra $g$ is sympathetic if $g=[g, g]$, $\operatorname{Der}(g)=\operatorname{ad}(g)$ and $Z(g)=\{0\}$.

(iii) Let $g$ a Lie algebra, and let $I$ an ideal of $g$.

$I$ is a direct factor of $g$ if there exists an ideal $J$ of $g$ such that $g=I \oplus J$.

(iv) A Lie algebra is irreducible if it has no non-zero direct factor.

\section{Introduction and principal results}

Let us give a brief survey of sympathetic Lie algebras story. In the seventies, M. Flato asked the following question: "Any semi-simple Lie algebra $g$ satisfies $[g, g]=g, \operatorname{Der}(g)=\operatorname{ad}(g), Z(g)=\{0\}$; are these three properties characteristic of semi-simple Lie algebras?".

A negative answer was given about twenty years later by $\mathrm{E}$. Angelopoulos [An], who constructed a class of Lie algebras satisfying these three properties, but which are not semi-simple, the counter-example of minimal dimension being a Lie algebra of dimension 35 and Levi subalgebra isomorphic to sl(2). We call the Lie algebras $g$ which satisfy $[g, g]=g, Z(g)=\{0\}$ and $\operatorname{Der}(g)=\operatorname{ad}(g)$ the sympathetic Lie algebras.

In [ABBP], we showed that this sympathetic Lie algebra $g$ of dimension 35 satisfies $H^{2}(g, g)=\{0\}$, which implies that it is (a) rigid (Lie algebra). Therefore the sympathetic structure isn't a direct degeneration of semisimple structure (e.g., by contraction). Moreover, for fixed dimension, sympathetic Lie algebras provide an open set in the variety of Lie algebras. So we started a general study of this structure. 
In [Be1], we showed that several classical properties of semi-simple Lie algebras are still valid for the class of sympathetic Lie algebras. For instance:

(i) Let $g$ be a sympathetic Lie algebra, $I$ an ideal of $g$. Then, $I$ is sympathetic if and only if $I$ is a direct factor of $g$ [Be1, corollary 2 of proposition 4].

(ii) Every extension of two sympathetic Lie algebras is sympathetic and trivial [Be1, corollary 3 of proposition 4].

(iii) A Lie algebra is sympathetic if and only if it is a direct sum of irreducible sympathetic ideals [Be1, theorem 1].

In [Be2], we give an example of a non semi-simple sympathetic Lie algebra of dimension 48 which is non rigid by deformations. And in [AnBe], we give the first examples of non semi-simple sympathetic Lie algebras with invariant scalar products (i.e. symmetric, non-degenerate and invariant bilinear forms).

In the present paper, we continue the study initiated in [Be1]. Actually, several results can be started for perfect Lie algebras, and then restricted to sympathetic ones.

In the first section of this paper, we recall Angelopoulos method of construction of non semi-simple sympathetic Lie algebras [An]. In the second section, we construct a non semi-simple sympathetic Lie algebra of dimension 25 . This Lie algebra is the smallest non semi-simple sympathetic Lie algebra known until now.

In the third section, we show that several classical properties of semisimple Lie algebras are still valid for the class of perfect Lie algebras. In this section, we obtain the following principal results:

(i) Let $g$ be a perfect Lie algebra.

Then $g=h_{1} \oplus \cdots \oplus h_{n}$ where each $h_{i}$ is an irreducible perfect ideal of $g$. Furthermore, this decomposition is unique.

This result generalizes theorem 1 of [Be1], and shows that the decomposition of a sympathetic Lie algebra into irreducible sympathetic ideals is unique.

(ii) Let $g$ be a sympathetic Lie algebra, and $g=h_{1} \oplus \cdots \oplus h_{n}$ be its unique decomposition into irreducible sympathetic ideals.

Then $h_{1} \ldots, h_{n}$ are the only irreducible sympathetic ideals of $g$. 


\section{Saïd Benayadi}

These results are classical if we replace perfect or sympathetic by semi-simple, but the semi-simplicity isn't a necessary condition to obtain them.

It is known that every Lie algebra $g$ has a greatest semi-simple ideal $S$, and that its radical $R(g)$ satisfies the following properties:

(i) $R(g) \cap S=\{0\}$;

(ii) $R(g)$ is the smallest among the ideals $I$ of $g$ for which $g / I$ is semisimple.

Then we ask the following questions:

Question 1 Does there exist a greatest sympathetic ideal $M$ of $g$ ?

Question 2 Does there exist a solvable ideal $P(g)$ of $g$ which is the greatest among solvable ideals $I$ of $g$ for which $I \cap M=\{0\}$ ?

Question 3 Does there exist an ideal $T(g)$ of $g$ which is the smallest among ideals $I$ of $g$ for which $g / I$ is a sympathetic Lie algebra?

Question 4 If $P(g)$ and $T(g)$ exist, do they coincide?

In the fourth section of this work, we answer to questions 1 and 2 , and in the fifth section we study the ideals $I$ of $g$ such that $g / I$ is a sympathetic Lie algebra. We obtain the following principal results:

(i) Let $g$ be a Lie algebra, $I$ and $J$ be two sympathetic ideals of $g$.

Then $I \cap J$ and $I+J$ are sympathetic ideals of $g$.

(ii) Every Lie algebra contains a greatest sympathetic ideal.

(iii) Let $g$ be a Lie algebra, $M$ its greatest sympathetic ideal. There exists a solvable ideal of $g$ denoted $P(g)$ which is the greatest among the solvable ideals $I$ of $g$ for which $I \cap M=\{0\}$.

(iv) $g$ is a sympathetic Lie algebra if and only if $P(g)=\{0\}$. If we replace sympathetic by semi-simple and $P(g)$ by $R(g)$, we obtain the characterization of semi-simple Lie algebras: $g$ is semi-simple if and only if $R(g)=\{0\}$. We call $P(g)$ the sympathetic radical of $g$.

(v) Let $g$ be a Lie algebra, $P(g)$ its sympathetic radical.

Then there exists a sympathetic subalgebra $m$ of $g$ such that $g=m \oplus P(g)$.

If we replace sympathetic by semi-simple and $P(g)$ by $R(g)$, we obtain the Levi-Malcev decomposition of $g$. 
(vi) Let $g$ be a Lie algebra, $H$ be an ideal of $g, C_{\infty}(g)$ be the union of the ascending central series of $g$. If $H$ is minimal among the ideals $I$ of $g$ for which $g / I$ is a sympathetic Lie algebra, then:

(1) $H$ is a solvable ideal of $g$,

(2) $C_{\infty}(g) \subseteq H \subseteq P(g)$.

In the sixth section, we give negative answers to questions 3 and 4 , giving examples of the following situation:

(i) Let $g$ be a Lie algebra, $I$ and $J$ be ideals of $g$ such that $g / I$ and $g / J$ are sympathetic Lie algebras. It can happen that $g / I \cap J$ is not a sympathetic Lie algebra.

(ii) There exists Lie algebras $g$ which have an infinite number of minimal ideals among the ideals $I$ of $g$ for which $g / I$ is a sympathetic Lie algebra.

Therefore, the answer to question 3 is no.

(iii) There exists Lie algebras $g$ such that $P(g)$ and $T(g)$ do exist as defined in questions 2 and 3 , but do not coincide.

Therefore, the answer to question 4 is no. Moreover:

(iv) There exists irreducible sympathetic Lie algebras with (non trivial) sympathetic non semi-simple quotients.

This last example shows that (contrarily to the semi-simple case) irreducibility, in the sympathetic case, is not really a minimal condition. One should introduce a smaller class of irreducible sympathetic Lie algebras without (non trivial) sympathetic non semi-simple quotients. For instance Angelopoulos's examples belong to this class.

In our opinion, these examples show how flabby the sympathetic structure is (compared e.g., to semi-simple), and that classification is probably impossible, even if the Levi subalgebra $s$ is specified, and even if $s$ is specified to be $\operatorname{sl}(2)$.

The Lie algebras envisaged in this work are of finite dimension over $\mathbf{K}=\mathbb{R}$ or $\mathbb{C}$. 


\section{Angelopoulos's theorem and construction of non semi-simple sympathetic Lie algebras [An]}

In this section $\mathbf{K}=\mathbb{C}$.

\subsection{E. Angelopoulos's theorem}

Theorem [An]. - Let $g$ be a Lie algebra, $g=S \oplus R(g)$ be its Levi decomposition, and $S=\bigoplus_{j=1}^{j=n} S_{j}$ be the decomposition of $S$ into simple ideals. If $g$ satisfies the following assertions:

(i) $R(g)$ is nilpotent and $R(g)=g_{1} \oplus g_{2} \oplus g_{3} \oplus g_{4}$ where the $g_{i}, 1 \leq i \leq 4$, are non zero simple $S$-modules such that:

$$
\left[g_{1}, g_{1}\right]=g_{3}, \quad\left[g_{1}, g_{2}\right]=g_{4}=\left[g_{2}, g_{2}\right]=\left[g_{1}, g_{3}\right]
$$

(ii) $g_{4}$ is a non trivial $S$-module,

(iii) any two modules of $S$-modules $g_{1}, g_{2}, g_{3}, g_{4}, S_{1}, \ldots, S_{N}$ are non isomorphic,

then $g$ is a non semi-simple sympathetic Lie algebra.

1.2 Method of construction of non semi-simple sympathetic Lie algebras

Let $S=g_{0}$ be a simple Lie algebra and let $\left(g_{i}\right)_{1<i<4}$ be four $S$-modules. If there exist non trivial morphisms of $S$-modules:

$$
\begin{gathered}
\tau_{11}: g_{1} \wedge g_{1} \rightarrow g_{3}, \quad \tau_{12}: g_{1} \otimes g_{2} \rightarrow g_{4}, \\
\tau_{13}: g_{1} \otimes g_{3} \rightarrow g_{4}, \quad \tau_{22}: g_{2} \wedge g_{2} \rightarrow g_{4},
\end{gathered}
$$

and if:

(i) $g_{1} \wedge g_{1} \wedge g_{1}$ doesn't contain any $S$-module isomorphic to $g_{4}$,

(ii) the $S$-module $g_{4}$ is non trivial,

(iii) the modules $g_{i}, 0 \leq i \leq 4$, are simple and any two of them are non isomorphic,

then there exists a product $[\cdot, \cdot]$ on $g=\bigoplus_{i=0}^{4} g_{i}$ such that $g$ equipped with $[\cdot, \cdot]$ is a non semi-simple sympathetic Lie algebra. 


\subsection{Construction in case where $s=\operatorname{sl}(2)$}

We designate by $D(n)$ the $\mathrm{sl}(2)$-module of dimension $2 n+1, n \in(1 / 2) \mathbb{N}$.

Let $g_{j} \simeq D\left(i_{j}\right), 1 \leq j \leq 4$, be four non trivial sl(2)-modules such that:

(1) $i_{j} \neq 1$ for all $j \in\{1,2,3,4\}$, and $i_{j} \neq i_{j^{\prime}}$ for all $j, j^{\prime} \in\{1,2,3,4\}$;

(2) $g_{1} \wedge g_{1}$ (resp. $g_{2} \wedge g_{2}$ ) contains a sl(2)-submodule isomorphic to $g_{3}$ (resp. $\left.g_{4}\right) ; g_{1} \otimes g_{2}$ and $g_{1} \otimes g_{3}$ contain some sl(2)-modules isomorphic to $g_{4}$;

(3) $g_{1} \wedge g_{1} \wedge g_{1}$ has no sl(2)-submodule isomorphic to $g_{4}$, then there exists a product $[\cdot, \cdot]$ on $g=\operatorname{sl}(2) \oplus\left(\bigoplus_{j=1}^{j=4} g_{j}\right)$ such that:

i) $\left[g_{1}, g_{1}\right]=g_{3},\left[g_{1}, g_{2}\right]=\left[g_{2}, g_{2}\right]=\left[g_{1}, g_{3}\right]=g_{4},\left[g_{4}, g_{i}\right]=\{0\}$ for all $i \in\{1,2,3,4\},\left[s, s^{\prime}\right]=\left[s, s^{\prime}\right]_{\mathrm{sl}(2)}$ for all $s, s^{\prime} \in \mathrm{sl}(2)$,

ii) $g$ equipped with $[\cdot, \cdot]$ is a non semi-simple sympathetic Lie algebra.

REMARK AND DEFINITION - - Let $g=\operatorname{sl}(2) \oplus\left(\bigoplus_{j=1}^{j=4} g_{j}\right)$ be the Lie algebra above. $\mathrm{sl}(2)$ is a Levi component of $g$, and $R(g)=\bigoplus_{1 \leq i \leq 4} g_{i}$. We call $g$ a Lie-Angelopoulos algebra.

Examples $[\mathrm{An}]$

$$
\left.\begin{array}{l}
g_{1}=D(2 k), \quad g_{2}=D(4 k+r), \\
g_{3}=D(4 k-1), \quad g_{4}=D(6 k-1) \\
0<k, \quad 0 \leq r \leq 4 k-1, \quad r \neq 2 k-1
\end{array}\right\}
$$

$$
\left.\begin{array}{l}
g_{1}=D(2 k+1), \quad g_{2}=D(4 k+r), \\
g_{3}=D(4 k+1), \quad g_{4}=D(6 k+1) \\
0<k, \quad 0 \leq r \leq 4 k+2, \quad r \neq 1, \quad r \neq 2 k+1
\end{array}\right\}
$$




\section{A non semi-simple sympathetic Lie algebra of dimension 25}

In this section $\mathbf{K}=\mathbb{C}$. Recall the method of calculation of ClebschGordan coefficients of [ABBP].

Let $s=\mathbb{C}[p, q]$ with the Poisson bracket:

$$
\{u, v\}=\frac{\partial u}{\partial p} \frac{\partial v}{\partial q}-\frac{\partial u}{\partial q} \frac{\partial v}{\partial p}=\Lambda^{i j} \partial_{i} u \partial_{j} v,
$$

and let $S^{k}$ be the space of homogeneous polynomials of degree $k . S^{2}$ is invariant by the Poisson bracket, isomorphic to $\operatorname{sl}(2)$, and $S^{2 n}, n \in(1 / 2) \mathbb{N}$ is a sl(2)-module for the adjoint action isomorphic to $D(n)$.

The Moyal *-produit defined by:

$$
\begin{aligned}
u * v & =u v+\sum_{r \geq 1} \frac{1}{2^{r} r !} \sum_{\substack{i_{1}, \ldots, i_{r} \\
j_{1}, \ldots, j_{r}}} \Lambda^{i_{1} j_{1}} \cdots \Lambda^{i_{r} j_{r}} \partial_{i_{1}, \ldots, i_{r}} u \partial_{j_{1}, \ldots, j_{r}} v \\
& =u v+\sum_{r \geq 1} \frac{1}{2^{r} r !} P_{r}(u, v)
\end{aligned}
$$

is an associative product, and for $X$ in $S^{2}=\operatorname{sl}(2)$, we have $\{x, \cdot\}=[x, \cdot]_{*}$.

We identify $D(n)$ and $S^{2 n}, D(p)$ and $S^{2 p}$, the map $u \otimes v \rightarrow u * v$ of $D(n) \otimes D(p)$ in $D(n) * D(p)$ is an isomorphism of $\operatorname{sl}(2)$-modules. Using the development of $u * v$, we get directly the reduction $D(n) \otimes D(p)=$ $\sum_{i=|n-p|}^{i=n+p} D(i)$, which gives the coefficients of Clebsch-Gordan.

Proposition 2.1. - There exists a Lie algebra $g=\sum_{i=0}^{i=4} g_{i}$ such that:

(i) $g_{0}$ is isomorphic to $\mathrm{sl}(2)$;

(ii) the only (non zero) commutators between $g_{i}$ and $g_{j}, i, j \geq 1$ are:

$$
\left[g_{1}, g_{1}\right]=g_{3}, \quad\left[g_{1}, g_{2}\right]=\left[g_{2}, g_{2}\right]=\left[g_{1}, g_{3}\right]=g_{4} ;
$$

(iii) $g_{1} \simeq D(3), g_{2} \simeq D(2), g_{3} \simeq D(3), g_{4} \simeq D(1)$ as $g_{0}$-modules.

Proof. - By the reduction $D(n) \otimes D(p)=\sum_{i=|n-p|}^{n+p} D(i)$, there exist non trivial morphisms of $g_{0}$-modules:

$$
\begin{array}{ll}
\tau_{11}: D(3) \wedge D(3) \rightarrow D(3), & \tau_{12}: D(3) \otimes D(2) \rightarrow D(1), \\
\tau_{22}: D(2) \wedge D(2) \rightarrow D(1), & \tau_{13}: D(3) \wedge D(3) \rightarrow D(1) .
\end{array}
$$


These morphisms and the action of $g_{0}$ on each $g_{i}$ define a skew-symmetric product $[\cdot, \cdot]$ on $g$ such that the assertion (ii) is verified.

It's easy to see that the Jacobi identity is verified on

$$
\left[\sum_{i=1}^{4} g_{i},\left[g_{0}, \sum_{i=1}^{4} g_{i}\right]\right] \text { and on }\left[g_{0},\left[g_{0}, \sum_{i=1}^{4} g_{i}\right]\right]
$$

Since $D(3) \wedge D(3) \wedge D(3)=D(6) \oplus D(4) \oplus D(3) \oplus D(2) \oplus D(0)$, then $g_{1} \wedge g_{1} \wedge g_{1}$ doesn't contain a $g_{0}$-submodule isomorphic to $g_{4}$, which implies that the Jacobi identity is verified on $\left[g_{1},\left[g_{1}, g_{1}\right]\right]$.

We conclude that $g$ with the product $[\cdot, \cdot]$ is a Lie algebra which verifies (i), (ii), and (iii).

Realizations of $g$. - We can realize $g$ as the space of homogeneous polynomials of even degree $\leq 6$ without constant terms. The bracket $[\cdot, \cdot]$ on $g$ is defined by:

(a) $g_{1}=\{(v, 0)\}$ and $g_{3}=\{(0, v)\}$ where $v \in D(3)$. Let $v, w \in D(3)$, i) $[(v, 0),(w, 0)]=\left(0, P_{3}(v, w)\right)$, we recall that $P_{3}(v, w)=$ - $P_{3}(w, v)$ because $P_{3}$ is skew-symmetric.

ii) $[(v, 0),(0, w)]=P_{5}(v, w)$

$$
[(0, v),(w, 0)]=-[(w, 0),(0, v)]=P_{5}(w, v)=-P_{5}(v, w)
$$

because $P_{5}$ is skew-symmetric. So $[(v, 0),(0, w)]=[(0, v),(w, 0)]$.

(b) Let $X, Y \in D(2)$,

$$
[X, Y]=P_{3}(X, Y) .
$$

(c) Let $X \in D(2)$ and $v \in D(3)$,

$$
[(v, 0), x]=P_{4}(v, x) \text { and }[x,(v, 0)]=-P_{4}(x, v),
$$

We recall that $P_{4}$ is symmetric.

(d) Let $i \in\{1,2,3,4\}, x \in g_{i}, s \in g_{0}$,

$$
[s, x]=s \cdot x .
$$

(e) $\left[s, s^{\prime}\right]=\left[s, s^{\prime}\right]_{S}$ for all $s, s^{\prime} \in g_{0}=S$, where $[\cdot, \cdot]_{S}$ is the bracket on $S=\operatorname{sl}(2)$. 
THEOREM 2.1.- $g$ is a non semi-simple sympathetic Lie algebra of dimension 25.

Proof. - It's easy to see that $R=\sum_{i=1}^{i=4} g_{i}$ is the radical of $g$, which implies that $g$ isn't semi-simple. Since $R$ doesn't contain any $g_{0}$-submodule isomorphic to $D(0)$, then $Z(g)=\{0\}$. $[g, g]=g$ because each $g_{i}$ is a non trivial simple $g_{0}$-module.

Let $D$ be a derivation of $g$, then there exists $y \in g$ such that $(D-$ $\operatorname{ad} y) / g_{0}=0$, which implies that $T=D-\operatorname{ad}(y)$ is a morphism of $g_{0^{-}}$ modules.

Therefore

$$
T\left(g_{0} \oplus g_{4}\right) \subseteq g_{0} \oplus g_{4}, \quad T\left(g_{1} \oplus g_{3}\right) \subseteq g_{1} \oplus g_{3}, \quad T\left(g_{2}\right) \subseteq g_{2}
$$

Since $[R, R]$ is a characteristic ideal of $g$ and $[R, R]=g_{3} \oplus g_{4}$, then $T\left(g_{3}\right) \subseteq g_{3}$ and $T\left(g_{4}\right) \subseteq g_{4}$.

By Schur's Lemma, we have

$$
T / g_{2}=\alpha \operatorname{id}\left(g_{2}\right), \quad T / g_{3}=\beta \operatorname{id}\left(g_{3}\right), \quad T / g_{4}=\gamma \operatorname{id}\left(g_{4}\right)
$$

and $T((v, 0))=a(v, 0)+b(0, v)$ for all $v \in D(3)$, where $\alpha, \beta, \gamma, a, b \in \mathbb{C}$. So we obtain:

$$
\left(\begin{array}{c}
\alpha+\beta=\gamma \\
a+\alpha=\gamma \\
2 \alpha=\gamma \\
2 a=\beta \\
b=0
\end{array}\right)
$$

This implies that $a=b=\alpha=\beta=\gamma=0$, therefore $T=0$. So $D=\operatorname{ad}(y) \in \operatorname{ad}(g)$.

We conclude that $g$ is a non semi-simple sympathetic Lie algebra.

Remark. - This Lie algebra is the smallest non semi-simple sympathetic Lie algebra known until now. Recall that 25 is the dimension of this Lie algebra.

Question 1.- J. Simon [Si] showed that there doesn't exist any non semi-simple sympathetic Lie algebra $g$ such that $\operatorname{dim} g \leq 10$. Does exist a non semi-simple sympathetic Lie algebra $g$ such that $11 \leq \operatorname{dim} g \leq 24$ ? 


\section{Decomposition of perfect Lie algebras}

Proposition 3.1. - Let $g$ be a perfect Lie algebra and let $I$ be an ideal of $g$. If $I$ is a direct factor of $g$, then $I$ is perfect.

Proof. - $I$ is a direct factor of $g$, then there exists $J$ an ideal of $g$ such that $g=I \oplus J$. Since $[I, J]=\{0\}$, then we have $[g, g]=[I, I] \oplus[J, J]$ consequently $I=[I, I]$.

Lemma 3.1. - Let $g$ be a Lie algebra. Then $g=I_{1} \oplus \cdots \oplus I_{n}$ where each $I_{i}$ is an irreducible ideal of $g$.

Proof. - We will prove the Lemma by induction on the dimension of $g$. If $\operatorname{dim} g=0$ or 1 , then the lemma is true.

We assume that the lemma is true if $\operatorname{dim} g<n$. Let $g$ be a Lie algebra such that $\operatorname{dim} g=n$. If $g$ isn't irreducible, then there exist two proper ideals of $g$ such that $g=I \oplus J$.

By our inductive hypothesis, we have $I=I_{1} \oplus \cdots \oplus I_{n}$ where each $I_{i}$ is an irreducible ideal of $I$ and $J=J_{1} \oplus \cdots \oplus J_{m}$ where each $J_{i}$ is an irreducible ideal of $J$.

To conclude, it is sufficient to see that every ideal of $I$ (resp. $J$ ) is an ideal of $g$.

LEMMA 3.2. - Let $g$ be a Lie algebra and let $I$ be a perfect ideal of $g$. If $g=I_{1} \oplus \cdots \oplus I_{n}$ where each $I_{i}$ is an ideal of $g$, then

$$
I=I \cap I_{1} \oplus \cdots \oplus I \cap I_{n} .
$$

Proof. - Given that $I$ is perfect, then $[I, g]=I$, consequently $I=$ $\left[I, I_{1}\right] \oplus \cdots \oplus\left[I, I_{n}\right]$. Which proves that $I=I \cap I_{1} \oplus \cdots \oplus I \cap I_{n}$.

THEOREM 3.1. - Let $g$ be perfect algebra. Then $g=h_{1} \oplus \cdots \oplus h_{n}$ where each $h_{i}$ is an irreducible perfect ideal of $g$. Furthermore, this decomposition is unique. 
Proof. - The existence of the decomposition results from Proposition 3.1 and Lemma 3.1. Let

$$
g=h_{1} \oplus \cdots \oplus h_{n}=h_{1}^{\prime} \oplus \cdots \oplus h_{m}^{\prime}
$$

be two decomposition of $g$ into irreducible perfect ideals.

Let $i \in\{1, \ldots, n\}$, by Lemma 3.2 , we have

$$
h_{i}=h_{i} \cap h_{1}^{\prime} \oplus \cdots \oplus h_{i} \cap h_{m}^{\prime},
$$

therefore there exist $\sigma(i) \in\{1, \ldots, m\}$ such that $h_{i}=h_{i} \cap h_{\sigma(i)}^{\prime}$, which implies that $h_{i} \subseteq h_{\sigma(i)}^{\prime}$. By Lemma 3.2,

$$
h_{\sigma(i)}^{\prime}=h_{1} \cap h_{\sigma(i)}^{\prime} \oplus \cdots \oplus h_{n} \cap h_{\sigma(i)}^{\prime},
$$

then there exists $j \in\{1, \ldots, m\}$ such that $h_{j} \cap h_{\sigma(i)}^{\prime} \neq\{0\}$, consequently $i=j$ and $h_{i}=h_{\sigma(i)}^{\prime}$.

Thus $n=m$ and there exists a permutation $\sigma$ of $\{1, \ldots, n\}$ such that $h_{i}=h_{\sigma(i)}^{\prime}$ for all $i \in\{1, \ldots, n\}$, so that the decomposition of $g$ into irreducible perfect ideals is unique.

CoRollaRY . - Let $g$ be a perfect Lie algebra, I be a proper ideal of $g$ and let $g=h_{1} \oplus \cdots \oplus h_{n}$ be the unique decomposition of $g$ into irreducible perfect ideal.

(i) $I$ is an irreducible direct factor of $g$ if and only if there exists $i \in\{1, \ldots, n\}$ such that $I=h_{i}$.

(ii) $I$ is a direct factor of $g$ if and only if there exists $\left\{i_{i}, \ldots, i_{m}\right\}$ a subset of $\{1, \ldots, n\}$ such that $I=h_{i_{1}} \oplus \cdots \oplus h_{i_{m}}$.

\section{Proof}

(i) Assume that $I$ is an irreducible direct factor of $g$, by Proposition 3.1 and Lemma 3.2 there exists $i \in\{1, \ldots, n\}$ such that $I=I \cap h_{i}$, so that $I \subseteq h_{i}$. Since $I$ is a direct factor of $g$, then there exists an ideal $J$ of $g$ such that $g=I \oplus J$, and by Lemma $3.2, h_{i}=h_{i} \cap I$, which implies that $I=h_{i}$.

(ii) Assume that $I$ is a direct factor of $g$, by Proposition 3.1, $I$ is a perfect ideal of $g$, and by Theorem 3.1 , there exist $I_{1}, \ldots, I_{m}$ irreducible perfect ideals of $I$ such that $I=I_{1} \oplus \cdots \oplus I_{m}$. 
To conclude, it is sufficient to see that every ideal of $I$ is an ideal of $g$ and to apply (i). The converse is evident.

LEMMA 3.3. - Let $g$ be a Lie algebra and I be a direct factor of $g$.

(i) If $Z(g)=\{0\}$, then $Z(I)=\{0\}$.

(ii) If $\operatorname{Der}(g)=\operatorname{ad}(g)$, then $\operatorname{Der}(I)=\operatorname{ad}(I)$.

Proof. - $I$ is a direct factor of $g$, then there exists $J$ an ideal of $g$ such that $g=I \oplus J$. The assertion (i) is true because

$$
Z(I) \subseteq Z(g)=\{0\} .
$$

(ii) Let $d \in \operatorname{Der}(I)$, we consider the endomorphism of $g$ defined by: $D / I=d$ and $D / J=0$, it is easy to check that $D$ is a derivation of $g$, therefore there exist $x \in g$ such that $D=\operatorname{ad}_{g}(x)$.

Since $g=I \oplus J$, then $x=x_{I}+x_{J}$ where $x_{I} \in I$ and $x_{J} \in J$, consequently $d=\operatorname{ad}_{I}\left(x_{I}\right)$. We get that $\operatorname{Der}(I)=\operatorname{ad}(I)$.

\section{Proposition 3.2}

(i) Let $g$ be a perfect Lie algebra such that $\operatorname{Der}(g)=\operatorname{ad}(g)$ (resp. $Z(g)=\{0\})$. Then

$$
g=h_{1} \oplus \cdots \oplus h_{n}
$$

where each $h_{i}$ is an irreducible perfect ideal of $g$ such that

$$
\operatorname{Der}\left(h_{i}\right)=\operatorname{ad}\left(h_{i}\right) \quad\left(\text { resp. } Z\left(h_{i}\right)=\{0\}\right) .
$$

Furthermore, this decomposition is unique.

(ii) Let $g$ be a sympathetic Lie algebra. Then

$$
g=h_{1} \oplus \cdots \oplus h_{n}
$$

where each $h_{i}$ is an irreducible sympathetic ideal of $g$.

Furthermore, this decomposition is unique.

From [Be1, corollary 2 of the proposition 1], corollary of Theorem 3.1 and Proposition 3.2 results the following corollary. 
Saïd Benayadi

CoROllary .- Let $g$ be a sympathetic Lie algebra.

(i) $g$ has a finite number of irreducible sympathetic ideals $h_{1}, \ldots, h_{n}$ and $g=h_{1} \oplus \cdots \oplus h_{n}$.

(ii) An ideal $I$ of $g$ is sympathetic if and only if $I=h_{i_{1}} \oplus \cdots \oplus h_{i_{m}}$ where $\left\{i_{1}, \ldots, i_{m}\right\} \subseteq\{1, \ldots, n\}$.

Proposition 3.3.- Let $g$ be a perfect Lie algebra and let $S$ be its greatest semi-simple ideal. Then there exists a perfect ideal $I$ of $g$ such that $g=S \oplus I$ and $I$ has no non-zero semi-simple ideal.

Proof. - Since $S$ is a semi-simple ideal of $g$, there exists $I$ an ideal of $g$ such that $g=S \oplus I$. By Proposition 3.1, I is a perfect ideal of $g$.

Let $J$ be a semi-simple ideal of $I$, then $J$ is a semi-simple ideal of $g$ so $J \subseteq I \cap S=\{0\}$.

Remark. - Proposition 3.3 reduces the study of perfect Lie algebras to those which have no non-zero semi-simple ideal. Combining with Theorem 3.1 , we see that such a Lie algebra is reduced to a direct sum of non-simple irreducible perfect ideals.

\section{The greatest sympathetic ideal and the sympathetic radical of Lie algebra}

THEOREM 4.1.- Let $g$ be a Lie algebra. If $I$ and $J$ are two ideals satisfying:

(i) I is a sympathetic ideal of $g$,

(ii) there exists a vector subspace $V$ of $g$ such that $g=J \oplus V$ and $[I, V] \subseteq V$. Then $I \cap J$ is a sympathetic ideal of $g$.

Proof.- Since $I=[I, I]$, we have $I=[I, g]$, consequently $I=$ $[I, J] \oplus[I, V]$. Then $[I, J]$ is a direct factor of $I$ because $[I, V]$ is an ideal of $I$.

By [Be1 corollary 2 of proposition 4$],[I, J]$ and $[I, V]$ are ideals of $g$, therefore $Z(I /[I, J])=\{0\}$. 
Structure of perfect Lie algebras without center and outer derivations

We consider the canonical surjection $\varphi: I \rightarrow I /[I, J]$. Let $x \in I \cap J$ and let $y \in I$,

$$
[\varphi(x), \varphi(y)]=\varphi([x, y])=0, \quad \text { so } x \in[I, J] .
$$

Thus $I \cap J=[I, J]$, consequently $I \cap J$ is a sympathetic ideal of $g$.

CoRollary 1. - Let $g$ be a Lie algebra, $I$ and $J$ be two ideals of $g$.

(i) If $I$ is a sympathetic ideal of $g$ and $J$ is a direct factor of $g$, then $I \cap J$ is a sympathetic ideal of $g$.

(ii) If $I$ and $J$ are sympathetic ideals of $g$, then $I \cap J$ and $I+J$ are sympathetic ideals of $g$.

Proof. - The assertion (i) results directly from Theorem 4.1.

(ii) By [Be1, corollary 1 of the proposition 4], we have $J$ is a factor direct of $g$, consequently $I \cap J$ is a sympathetic ideal of $g$. The following sequence is exact:

$$
0 \longrightarrow I \stackrel{\psi}{\longrightarrow} I+J \stackrel{\phi}{\longrightarrow} J / I \cap J \longrightarrow 0
$$

defined by $\psi(x)=x$ for all $x \in I$ and $\phi\left(x_{I}+x_{J}\right)=N\left(x_{J}\right)$ for all $x_{I} \in I$ and all $x_{J} \in J$ where $N: J \rightarrow J / I \cap J$ is the canonical surjection.

Thus $I+J$ is an extension of sympathetic Lie algebras, so $I+J$ is a sympathetic ideal of $g$ by [Be1, corollary 3 of the proposition 4].

CoRollary 2.- Every Lie algebra contains a greatest sympathetic ideal.

Proof. - Let $g$ be a Lie algebra.

$$
\mathcal{A}=\{\operatorname{dim} I, I \text { is a sympathetic ideal of } g\}
$$

is a non-empty subset of $\mathbb{N}$ bounded above by $\operatorname{dim} g$, then it has a greatest element $p$, consequently there exists a sympathetic ideal $M$ of $g$ such that $\operatorname{dim} M=p$.

Let $I$ be a sympathetic ideal of $g$; by corollary 1 of Theorem 4.1, $M+I$ is a sympathetic ideal of $g$. Thus $M=M+I$, so $I \subseteq M$.

We conclude that $M$ is the greatest sympathetic ideal of $g$. 
Lemma 4.1. - Let $g$ be a Lie algebra and let $I$ be an ideal of $g$. If there exist an ideal $J$ of $g$ satisfying:

(i) $[J, J]=J$ and $Z(J)=\{0\}$,

(ii) $g=I \oplus J$.

Then $I$ is a characteristic ideal of $g$.

Proof. - Let $x \in I$ and let $D \in \operatorname{Der}(g) . D(x)=y+z$ where $y \in I$ and $z \in J$. Let $z^{\prime} \in J$, then $\left[z, z^{\prime}\right]=-\left[x, D z^{\prime}\right]$. By Lemma 1 of $[\mathrm{Be} 1], J$ is a characteristic ideal of $g$, then $\left[z, z^{\prime}\right]=0$. Which proves that $z=0$, consequently $D(x) \in I$. We conclude that $D(I) \subseteq I$.

Proposition 4.1.- Let $g$ be a Lie algebra and $M$ be its greatest sympathetic ideal. Then there exists a characteristic ideal $L$ of $g$ satisfying:

(i) $g=M \oplus L$.

(ii) $L$ is the greatest among the ideals of $g$ which are direct factors of $g$ and do not have any non-zero sympathetic ideal.

Proof. - By [Be1, corollary 2 of the proposition 4], there exists an ideal $L$ of $g$ such that $g=M \oplus L$, and by Lemma 4.1, $L$ is a characteristic ideal of $g$.

Let $I$ be a sympathetic ideal of $L$, then $I$ is a sympathetic ideal of $g$, consequently $I \subseteq M \cap L=\{0\}$.

Let $J$ be an ideal of $g$ which is a direct factor of $g$ and doesn't have any non-zero sympathetic ideal, then there exists an ideal $H$ of $g$ such that $g=J \oplus H$.

Let $M^{\prime}$ be the greatest sympathetic ideal of $H$, then there exists an ideal $L^{\prime}$ of $H$ such that $H=M^{\prime} \oplus L^{\prime}$ and $L^{\prime}$ is a characteristic ideal of $H$ which doesn't have any non-zero sympathetic ideal.

Therefore, $g=M^{\prime} \oplus L^{\prime} \oplus J$ where $M^{\prime}$ is a sympathetic ideal of $g$ and where $L^{\prime}$ and $J$ are ideals of $g$ which don't have any non-zero sympathetic ideal. By [Be1, corollary 2 of proposition 4] and by Lemma 3.2, we get $M=M^{\prime}$.

Let $x \in J$, then $x=y+z$ where $y \in M$ and $z \in L$. Since $Z(M)=\{0\}$ and $[M, L+J]=\{0\}$, we have $x=z$. Then $J \subset L$. 
Definition 4.1. - Let g be a Lie algebra. Call sympathetic decomposition of $g$, the decomposition of the Proposition 4.1 (i.e. the decomposition $g=M \oplus L$ where $M$ is the greatest sympathetic ideal of $g$ and where $L$ is the greatest among the ideals of $g$ which are direct factors of $g$ and don't have any non-zero sympathetic ideal).

Proposition 4.2. - Let $g$ be a Lie algebra, $M$ be its greatest sympathetic ideal and $g=M \oplus L$ be its sympathetic decomposition. Then the following assertions are equivalent:

(i) $g$ is a sympathetic Lie algebra;

(ii) $L=\{0\}$;

(iii) $R(L)=\{0\}$.

Proof

(i) $\Rightarrow$ (ii) By [Be1, corollary 2 of proposition 4$], L$ is a sympathetic Lie algebra, so $L=\{0\}$.

(ii) $\Rightarrow$ (iii) It's clear.

(iii) $\Rightarrow($ i) $R(L)=\{0\}$ implies that $L$ is semi-simple, consequently $L=\{0\}$. Then $g=M$.

Definition 4.2. - Let $g$ be a Lie algebra, $M$ be its greatest sympathetic ideal and $g=M \oplus L$ be its sympathetic decomposition.

We call $R(L)$ the sympathetic radical of $g$ which will be denoted $P(g)$.

Remark. - This terminology is justified by Proposition 4.2.

Proposition 4.3. - Let $g$ be a Lie algebra, $M$ be its greatest sympathetic ideal and $P(g)$ be its sympathetic radical.

(i) $P(g)$ is a characteristic ideal of $g$.

(ii) $P(g)$ is the greatest among the solvable ideals $I$ of $g$ for which $I \cap M=\{0\}$.

(iii) There exists a sympathetic subalgebra $m$ of $g$ such that $g=m \oplus P(g)$.

Proof. - Let $g=M \oplus L$ be the sympathetic decomposition of $g$. The assertion (i) is clear. 
(ii) Let $I$ be a solvable ideal of $g$ such that $I \cap M=\{0\}$, then

$$
[M, I+P(g)]=\{0\},
$$

consequently $M \cap(I+P(g))=\{0\}$. This implies that $R(M) \cap(I+P(g))=$ $\{0\}$, therefore $I \subseteq P(g)$.

(iii) Let $S$ be a Levi component of $L$. By [Be1, corollary 3 of the proposition 4], $m=M \oplus S$ is a sympathetic Lie subalgebra of $g$. So that $g=m \oplus P(g)$ where $m$ is a sympathetic subalgebra of $g$.

Proposition 4.4.- Let $g$ be a Lie algebra, $P(g)$ be its sympathetic radical and $M$ be its greatest sympathetic ideal. If $m$ is a sympathetic subalgebra of $g$ such that $g=m \oplus P(g)$, then the following assertions are equivalent:

(i) $M \subseteq m$;

(ii) $[M, m] \subseteq m$;

(iii) $R(m)$ is an ideal of $g$.

Proof. - Let $g=M \oplus L$ be the sympathetic decomposition of $g$.

(i) $\Rightarrow$ (iii) $R(g)=R(m) \oplus P(g)$ implies that $R(m)=R(M)$.

(iii) $\Rightarrow$ (i) Let $S$ be a Levi component of $m$, then $S$ is a Levi component of $g$ and $S=S_{1} \oplus S_{2}$ where $S_{1}$ and $S_{2}$ are two ideals of $S$ such that $S_{1}$ (resp. $S_{2}$ ) is a Levi component of $M$ (resp. $L$ ). Thus $S_{1} \oplus R(m)$ is an ideal of $g$ such that $g=S_{1} \oplus R(m) \oplus L$, so that $M=S_{1} \oplus R(m)$. Which proves that $M \subseteq m$.

(ii) $\Rightarrow$ (i) $M=[M, M]$ implies that $[M, g]=M$, therefore $M=[m, M]$, which implies that $M \subseteq m$.

(i) $\Rightarrow$ (ii) It's clear.

Question 2. - Let $g$ be a Lie algebra. We know that if $S$ and $S^{\prime}$ are two Levi components of $g$, then there exists a special automorphism $\phi$ of $g$ such that $\phi(S)=S^{\prime}$ [Bo, Levi theorem]. So, if $m$ and $m^{\prime}$ are two sympathetic Lie subalgebras of $g$ satisfying

$$
g=m \oplus P(g)=m^{\prime} \oplus P(g),
$$

does there exist an automorphism $\varphi$ of $g$ such that $\varphi(m)=m^{\prime}$ ? 
The following proposition gives an answer in case where $m$ and $m^{\prime}$ contain the greatest sympathetic ideal $M$ of $g$.

Proposition 4.5. - Let $g$ be a Lie algebra, $P(g)$ its sympathetic radical, $M$ be its greatest sympathetic ideal and

$$
g=M \oplus L
$$

be its sympathetic decomposition. If $m$ and $m^{\prime}$ are two sympathetic Lie subalgebras $\psi$ of $g$ such that

$$
g=m \oplus P(g)=m^{\prime} \oplus P(g) \text { and } \quad M \subseteq m \cap m^{\prime},
$$

then there exists a special automorphism of $g$ such that $\psi(m)=m^{\prime}$.

Precisely, $\psi=e^{\operatorname{ad}_{g}(a)}$ where $a$ is an element of the nilpotent radical of $L$.

Proof. - By [Be1, corollary 2 of the proposition 4], $m=M \oplus S$ and $m^{\prime}=M \oplus S^{\prime}$ where $S$ (resp. $S^{\prime}$ ) is a semi-simple ideal of $m$ (resp. $m^{\prime}$ ).

By the Proposition 4.1, $L=S \oplus P(g)=S^{\prime} \oplus P(g)$ and, by [Bo, theorem 5 of sect. 6], there exists a special automorphism $e^{\operatorname{ad}_{L}(a)}$ of $L$ where $a$ is an element of nilpotent radical of $L$ such that $e^{\operatorname{ad}_{L}(a)}(S)=S^{\prime}$. Consequently, $\psi=e^{\operatorname{ad}_{g}(a)}$ is a special automorphism of $g$ such that $\psi(m)=m^{\prime}$.

Question 3 .- Let $g$ be a Lie algebra, $M$ be its greatest sympathetic ideal, and let $m$ be a sympathetic Lie subalgebra of $g$ such that $g=m \oplus P(g)$. Does $m$ contain $M$ ?

If the answer is affirmative, then the question 2 has the affirmative answer (prop. 4.5).

Lemma 4.2 [Bo]. - Let $g$ be a Lie algebra and $D \in \operatorname{Der}(g)$. If $D(R(g))=$ $\{0\}$, then $D$ is an inner derivation of $g$.

Proof. - Let $C$ be the centralizer of $R(g)$ in $g$. We consider the map

$$
\mathcal{S}: g / R(g) \longrightarrow \operatorname{gl}(C)
$$

defined by $\mathcal{S}(N(x))(y)=[x, y]$ for all $x \in g$ and all $y \in C$ where $N: g \rightarrow g / R(g)$ is the canonical surjection, this map is well defined and it's a representation of $g / R(g)$. 
Let $D \in \operatorname{Der}(g)$ such that $D(R(g))=\{0\}$, then the map $B: g / R(g) \rightarrow C$ defined by $B(N(x))=D(x)$ for all $x \in g$ is well defined and it's an element of $Z^{1}(g / R(g), C)$.

Since $g / R(g)$ is semi-simple, then $B \in B^{1}(g / R(g), C)$, consequently there exists $v \in C$ such that $B(N(x))=\mathcal{S}(N(x)) v$ for all $x \in g$. Thus $D=\operatorname{ad}_{g}(-v)$.

Proposition 4.6. - Let $g$ be a Lie algebra, $P(g)$ be its sympathetic radical and let $D \in \operatorname{Der}(g)$.

If $D(P(g))=\{0\}$, then $D$ is an inner derivation of $g$.

Proof. - Let $M$ be the greatest sympathetic ideal of $g$ and $g=M \oplus L$ the sympathetic decomposition of $g$.

- By [Be1, lemma 1], $M$ is a characteristic ideal of $g$, and by Proposition $4.1, L$ is a characteristic ideal of $g$, which implies that $D / M$ (resp. $D / L)$ is a derivation of $M$ (resp. $L)$.

Then there exists $x \in M$ such that $D / M=\operatorname{ad}_{M}(x)$, and by Lemma 4.2, there exists $y \in L$ such that $D / L=\operatorname{ad}_{L}(y)$. So $D=\operatorname{ad}_{g}(x+y)$.

Remark. - The Proposition 4.6 is an improvenent of Lemma 4.2 because $P(g) \subseteq R(g)$.

Proposition 4.7. - Let $g$ be a Lie algebra, $I$ and $J$ be two ideals of $g$, $P(I)$ and $P(J)$ be their sympathetic radicals.

If $I \cap J=\{0\}$, then $P(I) \oplus P(J)$ is the sympathetic radical of $I \oplus J$.

Proof. - Let $M_{1}$ (resp. $M_{2}$ ) be the greatest sympathetic ideal of $I$ (resp. $J$ ), and let $I=M_{1} \oplus L_{1}$ (resp. $J=M_{2} \oplus L_{2}$ ) be the sympathetic decomposition of $I$ (resp. $J$ ), then

$$
I \oplus J=\left(M_{1} \oplus M_{2}\right) \oplus\left(L_{1} \oplus L_{2}\right) .
$$

Let $M$ be the greatest sympathetic ideal of $I \oplus J$ and $I \oplus J=M \oplus L$ be the sympathetic decomposition of $I \oplus J$.

$[M, M]=M$ implies that $M=\left[M, M_{1} \oplus M_{2}\right]$ by [Be1, proposition 3] and by Proposition 4.1, we have

$$
M=M_{1} \oplus M_{2} \quad \text { and } \quad L=L_{1} \oplus L_{2},
$$

which proves that $P(I \oplus J)=P(I) \oplus P(J)$. 
Corollary . - Let $g, \ldots, g_{n}$ be Lie algebras, and let $P\left(g_{1}\right), \ldots, P\left(g_{n}\right)$ theirs sympathetic radicals. Then $P\left(g_{1}\right) \times \cdots \times P\left(g_{n}\right)$ is the sympathetic radical of $g_{1} \times \cdots \times g_{n}$.

Remark. - Let $g$ be a Lie algebra, $I$ be an ideal of $g$. We know that $R(I)=I \cap R(g)(\mathrm{cf} .[\mathrm{Bo}])$.

In the following proposition, we give a sufficient and necessary condition so that $P(I)=I \cap P(g)$.

Proposition 4.8 . - Let $g$ be a Lie algebra, $M$ be its greatest sympathetic ideal, $g=M \oplus L$ be its sympathetic decomposition, and let $I$ be an ideal of $g$.

$P(I)=I \cap P(g)$ if and only if $M \cap I$ is a sympathetic ideal of $g$.

Proof. - Let $M^{\prime}$ be the greatest sympathetic ideal of $I$ and let $I=$ $M^{\prime} \oplus L^{\prime}$ be the sympathetic decomposition of $I$.

$M^{\prime}=\left[M, M^{\prime}\right]$ implies that $M^{\prime}$ is a characteristic ideal of $I$ (by [Be1, lemma 1]), therefore $M^{\prime}$ is a sympathetic ideal of $g$, which implies that $M^{\prime} \subseteq M \cap I$.

Assume that $P(I)=I \cap P(g)$ and show that $M \cap I$ is a sympathetic ideal of $g$. Let $x \in M \cap I, x=z+y$ where $z \in M^{\prime}$ and $y \in L^{\prime}$, then

$$
x-z=y \in M \cap L^{\prime} .
$$

Let $S$ be a Levi component of $L^{\prime}$, then

$$
M \cap L^{\prime}=M \cap S \oplus M \cap P(I),
$$

consequently $M \cap L^{\prime}=M \cap S$. Then $M \cap L^{\prime}$ is a semi-simple ideal of $L^{\prime}$, therefore $M \cap L^{\prime}=\{0\}$. Thus $x=z \in M^{\prime}$. We conclude that $M \cap I=M^{\prime}$.

Conversely, we assume that $M \cap I$ is a sympathetic ideal of $g$ and we show that

$$
P(I)=I \cap P(g) .
$$

$M \cap I$ is a sympathetic ideal of $I$ implies that $M \cap I \subseteq M^{\prime}$, consequently

$$
M \cap I=M^{\prime} \text { and } M \cap L^{\prime}=\{0\} .
$$

Then $\left[M, L+L^{\prime}\right]=\{0\}$, therefore $M \cap\left(L+L^{\prime}\right)=\{0\}$, which implies that $L^{\prime} \subseteq L$. Thus $P(I)=P(g) \cap L^{\prime}$. We conclude that $P(I)=P(g) \cap I$ because $M^{\prime} \subseteq M$ and $L^{\prime} \subseteq L$. 


\section{Saïd Benayadi}

CoRollary .- Let $g$ be a Lie algebra, $M$ be its greatest sympathetic ideal and let $I$ be an ideal of $g$.

(i) If $I$ is a direct factor of $g$, then $P(I)=I \cap P(g)$.

(ii) If $M \subseteq I$, then $P(I)=I \cap P(g)$.

(iii) $\left[P\left(D^{n} g\right)\right]_{n \geq 0}$ and $\left[P\left(C^{n} g\right)\right]_{n \geq 1}$ are the decreasing sequences of solvable ideals of $g$.

(iv) $P\left(C^{n+1} g\right)$ contains $P\left(D^{n} g\right)$ for all $n \in \mathbb{N}$.

Proof

(i) Results from corollary 1 of Theorem 4.1 and Proposition 4.8.

(ii) Is a clear assertion.

To show (iii) and (iv), it is sufficient to see that $D^{n} g$ and $C^{n+1} g$ contain $M$ for all $n \in \mathbb{N}$ and to apply (ii).

\section{Ideals $I$ such that $g / I$ is a sympathetic Lie algebra}

Lemma 5.1. - Let $g$ be a Lie algebra and $I$ be an ideal of $g$ such that $Z(g / I)=\{0\}$. Then:

(i) $Z(g) \subseteq I$;

(ii) if $g=h_{1} \oplus \cdots \oplus h_{n}$ where each $h_{i}$ is an ideal of $g$, then $I=$ $I \cap h_{1} \oplus \cdots \oplus I \cap h_{n}$.

Proof. - We consider $\varphi: g \rightarrow g / I$ the canonical surjection.

(i) Let $x \in Z(g)$ and $y \in g$,

$$
[\varphi(x), \varphi(y)]=\varphi([x, y])=0
$$

then $\varphi(x) \in Z(g / I)=\{0\}$, which implies that $x \in I$. Hence $Z(g) \subseteq I$.

(ii) Let $x \in I$ and $y \in g$,

$$
x=x_{1}+\cdots+x_{n} \quad \text { and } \quad y=y_{1}+\cdots+y_{n}
$$

where $x_{i}, y_{i}$ are the elements of $h_{i}$ for all $i \in\{1, \ldots, n\}$. 
Structure of perfect Lie algebras without center and outer derivations

Let $i \in\{1, \ldots, n\}$,

$$
\left[\varphi\left(x_{i}\right), \varphi(y)\right]=\left[\varphi(x), \varphi\left(y_{i}\right)\right]=0,
$$

which implies that $\varphi\left(x_{i}\right) \in Z(g / I)=\{0\}$, therefore $x_{i} \in I \cap h_{i}$.

We conclude that $I=I \cap h_{1} \oplus \cdots \oplus I \cap h_{n}$.

LEMMA 5.2. - Let $g$ be a Lie algebra, $I$ and $J$ be ideals of $g$ such that $g=I \oplus J, I^{\prime}$ (resp. $\left.J^{\prime}\right)$ be an ideal of $I$ (resp. J). The following assertions are equivalent:

(i) $g /\left(I^{\prime} \oplus J^{\prime}\right)$ is a sympathetic Lie algebra;

(ii) $I / I^{\prime}$ and $J / J^{\prime}$ are sympathetic Lie algebras.

Proof. - Since $I$ and $J$ are direct factors of $g$, then $I^{\prime}$ and $J^{\prime}$ are ideals of $g$, so $I^{\prime} \oplus J^{\prime}$ is an ideal of $g$ and $g /\left(I^{\prime} \oplus J^{\prime}\right)$ is isomorphic to $I / I^{\prime} \oplus J / J^{\prime}$. Thus by [Be1, propositions 2 and 3], the assertions (i) and (ii) are equivalent.

Proposition 5.1. - Let $g$ be a Lie algebra, $I$ and $J$ be two ideals of $g$ such that $g=I \oplus J$ and let $H$ be an ideal of $g$. The following assertions are equivalent:

(i) $g / H$ is a sympathetic Lie algebra;

(ii) $I / I \cap H$ and $J / J \cap H$ are sympathetic Lie algebras.

\section{Proof}

(i) $\Rightarrow$ (ii) By Lemma 5.1, $H=H \cap I \oplus H \cap J$, and by Lemma 5.2, we have $I / I \cap H$ and $J / J \cap H$ are sympathetic Lie algebras.

(ii) $\Rightarrow$ (i) Let us show that

$$
H=H \cap I \oplus H \cap J .
$$

Indeed, let $x \in H$, then $x=y+z$ where $y \in I$ and $z \in J$. We consider the canonical surjections $\varphi: g \rightarrow g / H$ and $\phi: I \rightarrow I / I \cap H$. Let $y^{\prime} \in I$, then $\left[\varphi(y), \varphi\left(y^{\prime}\right)\right]=0$ which implies that $\left[y, y^{\prime}\right] \in H \cap I$, therefore $\left[\phi(y), \phi\left(y^{\prime}\right)\right]=0$.

Thus $\phi(y) \in Z(I / I \cap H)=\{0\}$, so that $y \in I \cap H$. By the same reasoning, we show that $z \in J \cap H$. We conclude that

$$
H=H \cap I \oplus H \cap J .
$$

So $g / H$ is a sympathetic Lie algebra by Lemma 5.2 . 
Proposition 5.2. - Let $g$ be a Lie algebra, $I$ and $J$ be two ideals of $g$ such that $g=I \oplus J$, and let $H$ be an ideal of $g$. The following assertions are equivalent:

(i) $H$ is minimal among the ideals $A$ of $g$ for which $g / A$ is a sympathetic Lie algebra;

(ii) $H \cap I$ (resp. $H \cap J$ ) is minimal among the ideals $A$ of $I$ (resp. $J$ ) for which $I / A$ (resp. J/A) is a sympathetic Lie algebra.

\section{Proof}

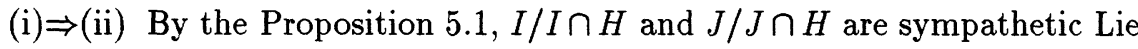
algebras. Let $I^{\prime}$ (resp. $J^{\prime}$ ) be an ideal of $I$ (resp. $J$ ) such that $I^{\prime} \subseteq I \cap H$ (resp. $J^{\prime} \subseteq J \cap H$ ) and $I / I^{\prime}$ (resp. $J / J^{\prime}$ ) is a sympathetic Lie algebra.

By Lemma 5.2, we have $g /\left(I^{\prime} \oplus J^{\prime}\right)$ is a sympathetic Lie algebra, consequently $H=I^{\prime} \oplus J^{\prime}$, and by Lemma 5.1, we conclude that $I^{\prime}=I \cap H$ and $J^{\prime}=J \cap H$.

(ii) $\Rightarrow$ (i) Let $H^{\prime}$ an ideal of $g$ such that $H^{\prime} \subseteq H$ and $g / H^{\prime}$ is a sympathetic

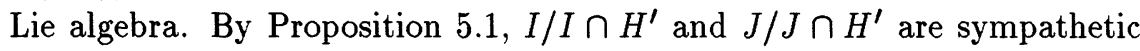
Lie algebras. Since $I \cap H^{\prime} \subseteq I \cap H$ and $J \cap H^{\prime} \subseteq J \cap H$, then we have $I \cap H^{\prime}=I \cap H$ and $J \cap H^{\prime}=J \cap H$, and by Lemma 5.1, $H=H^{\prime}$.

LEMMA 5.3. - Let $g$ be a Lie algebra, $\left(C_{n} g\right)_{n \geq 0}$ be the ascending central series of $g$, and let $C_{\infty}(g)$ be the union of the ascending central series of $g$.

Then, $C_{\infty}(g)$ is the smallest among the ideals $I$ of $g$ for which $Z(g / I)=$ $\{0\}$.

Proof. - There exists a positive integer $n$ such that $C_{\infty}(g)=C_{n}(g)$, then

$$
Z\left(g / C_{\infty}(g)\right)=C_{n+1}(g) / C_{n}(g)=\{0\} .
$$

Let $J$ be an ideal of $g$ such that $Z(g / J)=\{0\}$, by Lemma 5.1, $Z(g) \subseteq J$, i.e. $C_{1}(g) \subseteq J$. Assume that $C_{i}(g) \subseteq J$, and show that $C_{i+1}(g) \subseteq J$.

Indeed, $H=J / C_{i}(g)$ is an ideal of $A=g / C_{i}(g)$, and $A / H$ is isomorphic to $g / J$, which implies that $Z(A / H)=\{0\}$. Thus by Lemma 5.1 , we have $Z(A) \subseteq H$.

Consider the canonical surjection $f: g \rightarrow A$, then $J=f^{-1}(H)$ contain $C_{i+1}(g)=f^{-1}(Z(A))$. 
Structure of perfect Lie algebras without center and outer derivations

We conclude that $C_{i}(g) \subseteq J$ for all positive interger $i$, consequently $C_{\infty}(g) \subseteq J$.

TheOREM 5.1. - Let $g$ be a Lie algebra, $P(g)$ be its sympathetic radical and let $H$ be an ideal of $g$. If $H$ is minimal among the ideals $I$ of $g$ for which $g / I$ is a sympathetic Lie algebra, then:

(i) $H$ is solvable ideal of $g$,

(ii) $C_{\infty}(g) \subseteq H \subseteq P(g)$.

Proof. - $H=S \oplus R(H)$ where $S$ is a Levi component of $H$.

(i) We consider the canonical surjections $\varphi: g \rightarrow g / R(H)$ and $\psi: g \rightarrow g / H$. The following sequence is exact:

$$
0 \longrightarrow S \stackrel{\varphi_{\mid S}}{\longrightarrow} g / R(H) \stackrel{\phi}{\longrightarrow} g / H \longrightarrow 0
$$

where $\phi(\varphi(x))=\psi(x)$ for all $x \in g$.

Thus by [Be1, corollary 3 of the proposition 4$], g / R(H)$ is a sympathetic Lie algebra, so $R(H)=H$.

(ii) Let $M$ be the greatest sympathetic ideal of $g$ and $g=M \oplus L$ be the sympathetic decomposition of $g$.

By Proposition 5.2, $M \cap H$ is minimal among the ideals $A$ of $M$ for which $M / A$ is a sympathetic Lie algebra, which implies that $M \cap H=\{0\}$. Thus by Lemma 5.1 , we have $H=H \cap L$, so that $H \subseteq P(g)$.

Proposition 5.3. - Let g be a Lie algebra, $M$ be its greatest sympathetic ideal, $g=M \oplus L$ be its sympathetic decomposition.

If $L$ is solvable (i.e. $L=P(g)$ ), then $L$ is the smallest among the ideals $I$ of $g$ for which $g / I$ is a sympathetic Lie algebra.

Proof. - Let $H$ be an ideal of $g$ such that $g / H$ is a sympathetic Lie algebra, then there exists $H^{\prime}$ an ideal of $g$ such that $H^{\prime} \subseteq H$ and $H^{\prime}$ is minimal among the ideals $I$ of $g$ for which $g / I$ is a sympathetic Lie algebra. $g / H^{\prime}$ is isomorphic to $M \oplus L / H^{\prime}$, which implies that $L / H^{\prime}$ is a sympathetic and solvable Lie algebra, consequently we have $L=H^{\prime}$.

Proposition 5.4. - Let g be a Lie algebra, $M$ be its greatest sympathetic ideal, $P(g)$ be its sympathetic radical, $g=M \oplus L$ be its sympathetic decomposition and let $S$ be a Levi component of $L$. 
If $J$ is an ideal of $g$ such that:

(i) $g / J$ is a sympathetic Lie algebra,

(ii) $[S, P(g)] \subseteq J \subseteq P(g)$,

then $J=P(g)$.

Proof. - $S$ is a Levi component of $L$, then $g=S \oplus I$ where $I=M \oplus P(g)$. We consider the canonical surjection $\varphi: g \rightarrow g / J$, then we have $\varphi(g)=$ $\varphi(S) \oplus \varphi(I)$. Furthermore, $[S, P(g)] \subseteq J$ implies that $\varphi(S)$ is a semisimple ideal of $\varphi(g)$.

Since $\varphi(g)$ is sympathetic, $\varphi(I)$ is a sympathetic ideal of $\varphi(g)$, consequently $I / J$ is a sympathetic Lie algebra.

Thus by Proposition 5.3, $P(g) \subseteq J$, so that $P(g)=J$.

Proposition 5.5.- Let $g$ be a Lie algebra such that $[g, g]$ is a sympathetic Lie ideal of $g$, and let $P(g)$ be the sympathetic radical of $g$. Then:

(i) $g=Z(g) \oplus[g, g]$,

(ii) $[g, g]$ is the greatest sympathetic ideal of $g$,

(iii) $C_{\infty}(g)=Z(g)=P(g)$.

Proof

(i) The corollary 2 of the proposition 4 of [Be1] implies the existence of an ideal $I$ of $g$ such that $g=[g, g] \oplus I$.

$[I, g]=\{0\}$ implies that $Z(g)$ contains $I$, which yields $Z(g)=I$ because

$$
Z(g) \cap[g, g] \subseteq Z([g, g])=\{0\}
$$

(ii) Let $M$ be the greatest sympathetic ideal of $g . M=[M, M]$ implies that $M \subseteq[g, g]$, therefore $M=[g, g]$.

(iii) By Lemma 5.1 and by Lemma $5.3, C_{\infty}(g)=Z(g)$, and by (i), $P(g)=Z(g)$. 
Remarks

(1) The assertions (i) of Proposition 5.5 is an amelioration of the known result: if $g$ is a reductive Lie algebra (i.e. $[g, g]$ is semi-simple), then $g=Z(g) \oplus[g, g]$.

(2) If we replace sympathetic by semi-simple and $P(g)$ by $R(g)$ in the Proposition 5.5, the assertions (ii) and (iii) are classical when $g$ is reductive.

Question 4. - Let $g$ be a Lie algebra, $I$ and $J$ be ideals of $g$. If $g / I$ and $g / J$ are sympathetic Lie algebras, is $g / I \cap J$ a sympathetic Lie algebra?

\section{Counter-examples}

In this section $\mathbf{K}=\mathbb{C}$.

First counter-example

Let

$$
g=\operatorname{sl}(2) \oplus\left(\bigoplus_{1 \leq i \leq 4} g_{i}\right), \quad g^{\prime}=\operatorname{sl}(2) \oplus\left(\bigoplus_{1 \leq i \leq 4} g_{i}^{\prime}\right)
$$

be two Angelopoulos-Lie algebras. We assume that $g_{i}$ is not isomorphic to $g_{j}^{\prime}$ for all $i, j \in\{1,2,3,4\}$. Let $\Pi=\mathcal{S} / R\left(g^{\prime}\right)$ where $\mathcal{S}=\operatorname{ad} / \operatorname{sl}(2)$. $\Pi$ is a representation of $\operatorname{sl}(2)$ in $R\left(g^{\prime}\right)$ and $\Pi(s)$ is a derivation of $R\left(g^{\prime}\right)$ for all $s \in \operatorname{sl}(2)$.

Let $\widetilde{\Pi}: g \rightarrow \operatorname{gl}\left(R\left(g^{\prime}\right)\right)$ defined by: $\widetilde{\Pi}(s)=\Pi(s)$ for all $s \in \operatorname{sl}(2)$ and $\widetilde{\Pi}(r)=0$ for all $r \in R(g)$. Then, $\widetilde{\Pi}$ is a representation of $g$ in $R\left(g^{\prime}\right)$, and $\widetilde{\Pi}(x) \in \operatorname{Der}\left(R\left(g^{\prime}\right)\right)$.

We consider the semi-direct product

$$
h=g \times_{\widetilde{\Pi}} R\left(g^{\prime}\right) .
$$

Since $\left[\mathrm{sl}(2), g_{i}\right]=g_{i}$ and $\left[\operatorname{sl}(2), g_{i}^{\prime}\right]=g_{i}^{\prime}$ for all $i \in\{1,2,3,4\}$, then $[h, h]=h$.

$Z(h)=\{0\}$ because $g_{i} \not \neq D(0)$ and $g_{i}^{\prime} \not \neq D(0)$ for all $i \in\{1, \ldots, 4\}$. Let $D \in \operatorname{Der}(h)$. We may assume $D / \operatorname{sl}(2)=0$, then $D$ is a $\operatorname{sl}(2)$-morphism. Which implies that $D / g_{i}=\lambda_{i} \operatorname{id}\left(g_{i}\right)$ and $D / g_{i}^{\prime}=\alpha_{i} \operatorname{id}\left(g_{i}^{\prime}\right)$ where $\lambda_{i}, \alpha_{i} \in \mathbb{C}$, consequently $D / g \in \operatorname{Der}(g)$ (resp. $D / g^{\prime} \in \operatorname{Der}\left(g^{\prime}\right)$ ). 


\section{Saïd Benayadi}

Since $g$ and $g^{\prime}$ satisfy (i) of Theorem 1 (E. Angelopoulos), $D=0$. Therefore $h$ is a sympathetic Lie algebra.

Since sl(2) is a Levi component of $h$, then by Theorem $1, h$ is irreducible.

We consider $R(g)$ (or $R\left(g^{\prime}\right)$ ) which is an ideal of $h$, we have $h / R(g) \simeq g^{\prime}$ sympathetic Lie algebra.

We conclude that there exists an irreducible sympathetic Lie algebra which has (non trivial) non semi-simple sympathetic quotients.

Second counter-example

Let

$$
g=\operatorname{sl}(2) \oplus\left(\bigoplus_{1 \leq i \leq 4} g_{i}\right)
$$

be an Angelopoulos-Lie algebra, $V \simeq D(i)$ such that $i \neq 0,1$ and $g_{j} \not 2 D(i)$ for all $j \in\{1,2,3,4\}$.

Let $\Pi: \operatorname{sl}(2) \rightarrow \operatorname{gl}(V)$ the representation of $\operatorname{sl}(2)$ which is associated to the sl(2)-module $V$.

We consider $\widetilde{\Pi}: g \rightarrow \operatorname{gl}(V)$ defined by: $\widetilde{\Pi}(s)=\Pi(s)$ for all $s \in \operatorname{sl}(2)$, and $\widetilde{\Pi}(r)=0$ for all $r \in R(g)$, then $\widetilde{\Pi}$ is a representation of $g$ in $V$.

We consider the semi-direct product $h=g \times \widetilde{\Pi} V$ ( $V$ is a commutative Lie algebra). Since $\left[\operatorname{sl}(2), g_{i}\right]=g_{i}$ and $[\operatorname{sl}(2), V]=V$, then $[h, h]=h$. Moreover, $Z(g)=\{0\}$ because $g_{i} \not \neq D(0)$ for all $i \in\{1,2,3,4\}$ and $V \not D(0)$.

Let $D: h \rightarrow h$ defined by $D / \operatorname{sl}(2)=0, D / R(g)=0, D / V=\lambda \operatorname{id}(V)$, $\lambda \in \mathbb{C}-\{0\}$.

It's easy to check that $D \in \operatorname{Der}(h)$, and $D \notin \operatorname{ad}(h)$. So $h$ isn't a sympathetic Lie algebra.

sl(2) is a Levi component of $h$ and $R(h)=\bigoplus_{1 \leq i \leq 4} g_{i} \oplus V$. If $I$ is an ideal of $h$, then $I=h$ or $I$ is solvable. Thus $h$ has no sympathetic ideal, therefore $P(h)=R(h)$.

Since $V$ is a commutative ideal of $g$ and $h / V \simeq g$ is a sympathetic Lie algebra, $T(h)=V$. Consequently $T(h) \neq P(h)$. We conclude that there exists a Lie algebra $g$ such that $P(g)$ and $T(g)$ do exist as defined in questions 2 and 3 of introduction, but do not coincide.

Therefore, the answer to question 4 of introduction is no. 
Structure of perfect Lie algebras without center and outer derivations

\section{Third counter example}

We can improve the second counter-example by taking $V \simeq g_{4}$.

Every sl(2)-submodule of $g_{4} \oplus V$ is an ideal of $g$ (because $\left[\bigoplus_{1 \leq i \leq 4} g_{1}\right.$, $\left.\left.g_{4} \oplus V\right]=\{0\}\right)$.

Let $W$ be a proper sl(2)-submodule of $g_{4} \oplus V$, then $h / W$ is sympathetic (choose $W$ such that $W \cap g_{4}=\{0\}$, which is possible in infinitely many ways) and $W$ is minimal among the ideals $I$ of $g$ for which $g / I$ is a sympathetic Lie algebra.

Then, usually there doesn't exist a smallest ideal such that the quotient is a sympathetic Lie algebra.

Therefore, the answer to question 3 of introduction is no.

Taking $W_{1}$ and $W_{2}$ satisfying the hypothesis above, $W \neq W_{2}$ then $h / W_{1}$ and $h / W_{2}$ are sympathetic Lie algebras and $W_{1} \cap W_{2}=\{0\}$. Which proves that $h / W_{1}$ and $h / W_{2}$ are sympathetic Lie algebras, and $h / W_{1} \cap W_{2}=h$ isn't a sympathetic Lie algebra.

Which answers to question 4 (p. 229).

\section{Acknowledgements}

I should like to thank Professors M. Flato and G. Pinczon for suggesting me this subject and encouraging me and advising during its development. I also want to express my tanks to E. Angelopoulos for interesting discussions.

\section{References}

[An] Angelopoulos (E.) . - Algèbres de Lie satisfaisant $g=[g, g], \operatorname{Der}(g)=\operatorname{ad} g$, C.R. Acad. Sci. Paris, 306 (1988), pp. 523-525.

[AnBe] Angelopoulos (E.) and Benayadi (S.) .- Constructions d'algèbres de Lie sympathiques non semi-simples munies de produits scalaires invariants, C.R. Acad. Sci. Paris, 317 (1993), pp. 741-744.

[ABBP] Arnal (D.), Benamor (H.), Benayadi (S.) and Pinczon (G.) .- Une algèbre de Lie non semi-simple vérifiant $H^{1}(g)=H^{2}(g)=H^{0}(g, g)=H^{1}(g, g)=$ $H^{2}(g, g)=\{0\}$, C.R. Acad. Sci. Paris, 315 (1992), pp. 261-263.

[Be1] BenAYADI (S.) .- Certaines propriétés d'une classe d'algèbres de Lie qui généralisent les algèbres de Lie semi-simples. Ann. Fac. Sci. Toulouse, Vol. XII, 1 (1991), pp. 29-35.

[Be2] Benayadi (S.) Thèse, Université de Bourgogne 1993.

[Bo] Bourbaki (N.) .- Groupes et algèbres de Lie, Hermann, Paris, chap. 1 (1971).

[Si] SIMON (J.) Private communication. 